\title{
Factors associated with poor-to-moderate quality of life among pregnant women with gestational diabetes mellitus: a cross-sectional study in Malaysia
}

\begin{abstract}
Purpose: This study aimed to identify factors associated with poor-to-moderate quality of life (QOL) among women with gestational diabetes mellitus (GDM) in two tertiary hospitals in Malaysia. Methods: A cross-sectional study was conducted among 526 pregnant women with GDM in two tertiary hospitals in Malaysia. Diabetes-related QOL was assessed using the Asian Diabetes Quality of Life Scale (AsianDQoL). Socio-demographic characteristics, glucose monitoring treatments for GDM, past obstetric history, concurrent medical problems and a family history of diseases were captured from patient records. A multiple logistic regression was used for analysis. Results: A total of 526 respondents with GDM entered the analysis. The median age of the respondents was 32 (interquartile range $=7$ ) while $82.3 \%$ were Malay women. More than half of the respondents $(69.5 \%)$ received an oral hypoglycaemic agent (OHA), and/or diet modification in controlling their GDM. The study reported that $23.2 \%$ of the respondents had poor-to-moderate QOL. Those with a family history of depression and/or anxiety (adjusted Odds ratio [AOR] 6.934, 95\% confidence interval [CI] 2.280-21.081), and a family history of GDM (AOR 1.814, 95\% CI 1.185-2.778) were at higher odds of suffering from poor-to-moderate QOL compared to those without a family history. Similarly, those who received insulin, with or without OHA, and/or are on diet modification (AOR 1.955, 95\% CI 1.243-3.074) were at higher odds of suffering from poor-to-moderate QOL compared to those receiving OHA and/or diet modification. Conclusion: Nearly one-quarter of Malaysian women with GDM have poor-to-moderate QOL. GDM women with a family history of depression and/or anxiety, family history of GDM, and those who received insulin, with or without OHA, and/or are on diet modification were associated with poor-tomoderate QOL.
\end{abstract}

Keyword: Anxiety; Depression; Gestational diabetes mellitus; Malaysia; Quality of life 